\title{
Synergism between Bacterial GAPDH and OMVs: Disparate Mechanisms but Co-operative Action
}

\author{
David E. Whitworth * and Bethan H. Morgan \\ Institute of Biological, Environmental and Rural Sciences, Aberystwyth University, Aberystwyth, UK
}

Keywords: fusogen, extracellular vesicles, Myxococcus xanthus, secretion, virulence, pathogenesis

\section{INTRODUCTION}

Outer membrane vesicles (OMVs) shed from bacteria contribute to pathogenesis by promoting colonization of host tissues and trafficking virulence factors into host cells via fusion with the host cell plasma membrane. Glyeraldehyde-3-phosphate dehydrogenase (GAPDH) is also secreted by prokaryotes, but enhances pathogenesis by promoting adhesion of bacteria to host cell surfaces. However, GAPDH is also known to catalyze the fusion of membranes, and it has been shown to promote OMV activity in the non-pathogen Myxococcus xanthus. We suggest that during infection by Gram-negative bacteria, GAPDH and OMVs work synergistically to stimulate pathogenesis.

\section{OPEN ACCESS}

Edited by:

Miguel A. Aon

Johns Hopkins University School of Medicine, USA

Reviewed by:

Alberto A. Iglesias, Instituto de Agrobiotecnología del Litoral (UNL-CONICET), Argentina Catrin Ffion Williams,

Cardiff University, UK

${ }^{*}$ Correspondence: David E. Whitworth dew@aber.ac.uk

Specialty section:

This article was submitted to Microbial Physiology and Metabolism, a section of the journal

Frontiers in Microbiology

Received: 23 September 2015

Accepted: 20 October 2015

Published: 09 November 2015

Citation:

Whitworth DE and Morgan BH (2015) Synergism between Bacterial GAPDH and OMVs: Disparate Mechanisms but Co-operative Action.

Front. Microbiol. 6:1231.

doi: 10.3389/fmicb.2015.01231

\section{OUTER MEMBRANE VESICLES IN HEALTH AND DISEASE}

A common bacterial mechanism for engineering the environment involves the secretion of OMVs-10-300 nm diameter packages, pinched off from the outer membrane of Gram-negative bacteria, enclosing periplasmic material (Figure 1). OMV constituents can be specifically targeted for inclusion in OMVs, however the mechanisms of OMV biogenesis and cargo targeting remain poorly defined (Kulkarni and Jagannadham, 2014).

OMVs are able to migrate away from their producing cells, accessing niches unavailable to the producing cell, and delivering secreted material to distant sites of action. Packaging within OMVs means their contents are not diluted as they are transported far from the cell, are protected from the environment (e.g., extracellular proteases), and cargo complexes can be secreted as pre-assembled entities (Ellis and Kuehn, 2010; Kulkarni and Jagannadham, 2014). At their site of action, OMVs can deliver their contents by two mechanisms. They can fuse with target membranes (Figure 1; Kadurugamuwa and Beveridge, 1999; Bomberger et al., 2009), or contact with a surface can trigger OMV lysis (Kadurugamuwa and Beveridge, 1996), releasing OMV contents.

OMVs are produced by all Gram-negative bacteria, and are known to have diverse antimicrobial, biofilm-promoting, virus-resistance, quorum-signaling and virulence-enhancing properties (Manning and Kuehn, 2013). The virulence of pathogens is known to correlate with the degree of vesiculation (Rolhion et al., 2005), and OMVs are able to enhance colonization of host tissues, modify host cell biology, and/or protect the OMV-producer from therapeutics and the host immune response (Inagaki et al., 2006; Thay et al., 2014; Vanhove et al., 2015).

OMV production is induced by stresses associated with host colonization (McBroom and Kuehn, 2007), for example by exposure to host muscle tissue (Dutson et al., 1971). They are able to adhere to host cells (Inagaki et al., 2006), and promote biofilm formation in clinically important bacteria (Grenier and Mayrand, 1987; Kamaguchi et al., 2003; Yonezawa et al., 2009). The OMVs of many pathogens have been documented to contain toxins and other virulence factors (Elluri et al., 2014; Roier et al., 2014; Thay et al., 2014; Vanhove et al., 2015), and OMV-packaging has been shown to stabilize, activate and/or regulate toxin activity (Fahie et al., 2013; Bielaszewska et al., 2014; Elluri et al., 2014). 


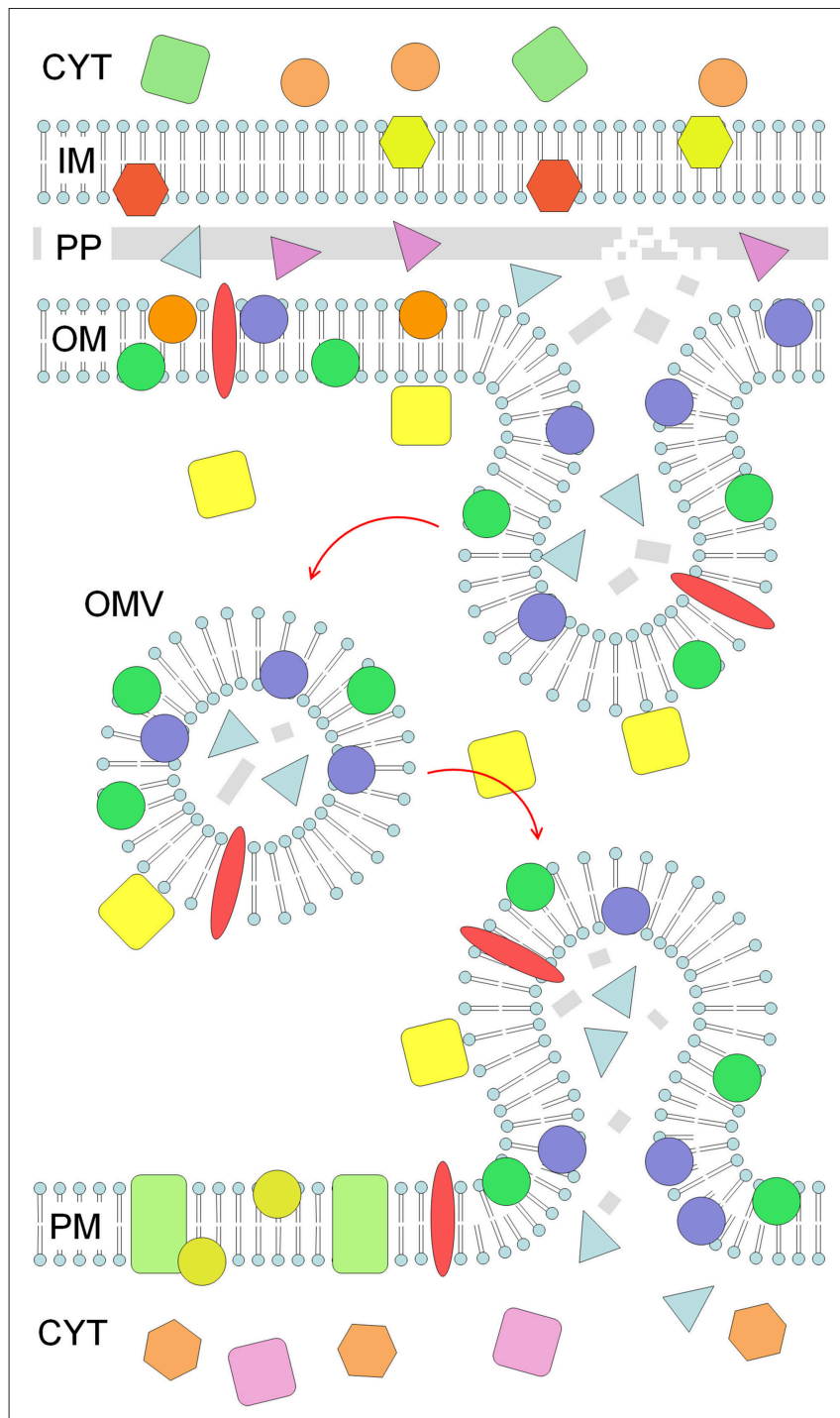

FIGURE 1 | OMV production and targeting to a eukaryotic cell. A Gram-negative cell (top) produces an OMV (middle) by pinching-off a protrusion of the outer membrane $(\mathrm{OM})$. The OMV is enriched in a subset of $\mathrm{OM}$ and periplasmic (PP) material, including specific proteins and peptidoglycan fragments (gray), while inner membrane (IM) and cytoplasmic (CYT) material is absent. The OMV is able to fuse with a target membrane (bottom), in this case the plasma membrane (PM) of a eukaryotic cell, delivering its contents into the PM and cytoplasm (CYT). GAPDH (yellow squares) is found on the surface of cells and OMVs, and can stimulate the fusion of OMVs with target membranes.

\section{THE GIFTED ENZYME GLYCERALDEHYDE-3-PHOSPHATE DEHYDROGENASE}

GAPDH (EC 1.2.1.12) is first encountered by biology students as an essential enzyme of central metabolism. It is a highly conserved protein, typically found as a tetramer (Seidler, 2013), and can be post-translationally modified in multiple ways (Sirover, 2014).
Intriguingly, GAPDH has been ascribed many additional roles beyond metabolism in eukaryotes, including glycosylation of uracil in DNA, transcriptional activation and apoptotic regulation (Sirover, 2005). One of its more exotic "moonlighting" activities is the ability to fuse membranes together (Glaser and Gross, 1995). This can occur in vitro, but has also been implicated in the fusion of secretory granules with the plasma membrane in neutrophils, fusion of presynaptic vesicles with the synaptic membrane (and their loading with cargo), axoplasmic transport, ER-Golgi vesicular shuttling, and nuclear membrane fusion (Glaser and Gross, 1995; Hessler et al., 1998; Ikemoto et al., 2003; Nakagawa et al., 2003). The structural basis of fusogenesis is unknown, however fusion requires binding to the relatively scarce membrane lipid phosphatidylserine (PS), and the PS binding site of GAPDH has been elucidated (Kaneda et al., 1997).

The classic glycolytic role of GAPDH places it in the cytoplasm, and it lacks an N-terminal signal sequence or other trafficking motif. However, with the advent of proteomics, many studies have identified GAPDH in extracellular fractions of a wide range of bacteria (Curtis et al., 2007; Holland et al., 2010; Deng et al., 2012; Vanden Bergh et al., 2013; Wang et al., 2013). It is a major surface protein of Gram-positive (Pancholi and Fischetti, 1992; Pasztor et al., 2010; Oliveira et al., 2012), and Gram-negative bacteria (Egea et al., 2007; Gao et al., 2014). In streptococci its release beyond the cell involves autolysis, with released protein then specifically binding to the surface of unlysed cells (Terrasse et al., 2015). Thus, GAPDH seems to be an almost ubiquitous protein, being commonly found within cells, on cells and beyond cells.

Extracellular bacterial GAPDH promotes adhesion to and invasion of host tissue, inhibits host lysozyme, and triggers apoptosis in macrophages (Seidler and Seidler, 2013). During host colonization, it is known to adhere to a variety of substrates, including PS, mucin, plasminogen and fibrinogen (Alvarez et al., 2003; Egea et al., 2007; Gao et al., 2014). It is likely that further mechanisms exist by which GAPDH promotes virulence, but studies have been hampered by difficulties in deleting the gene encoding GAPDH, due to its essential role in energy metabolism (Henderson and Martin, 2011).

\section{OMVS AND GAPDH WORKING TOGETHER}

The soil-dwelling myxobacterium $M$. xanthus is a predator of a wide range of bacteria and fungi, and OMVs are implicated in several aspects of its life-cycle (Whitworth, 2011). Its OMVs are loaded with hydrolases and they are able to kill other microbes, including Escherichia coli and Pseudomonas aeruginosa (Evans et al., 2012). Adding GAPDH to $M$. xanthus OMVs enhances their ability to kill prey cells. This is attributable to the fusogenic activity of the enzyme, as only intact OMVs exhibit cytotoxic activity (Evans et al., 2012), and OMVs of other bacteria are known to kill prey cells through fusion with their outer membrane (Kadurugamuwa and Beveridge, 1996). GAPDH has been found to be a major component of $M$. xanthus cells, OMVs and soluble secretome (Whitworth et al., 2015) suggesting GAPDH stimulates the antimicrobial activity of $M$. 
xanthus OMVs in the wild, by promoting their fusion with prey cells.

EVpedia, the EV database (Kim et al., 2013), shows that GAPDH has been observed as a component of the OMVs of many organisms (including E. coli, P. aeruginosa, Edwardsiella tarda, Francisella tularensis, Francisella philomiragia, Acinetobacter baumanii, and Neiserria meningiditis). Given the virtual ubiquity of GAPDH and OMV secretion, it is possible they could be working together in other contexts. The bacterial behavior for which there is most evidence of potential GAPDHOMV synergy is pathogenesis. If the activities analogous to those observed for $M$. xanthus occur for pathogens in vivo, then pathogen OMVs would be stimulated to fuse with target cells/membranes by pathogen-derived GAPDH.

\section{GAPDH/OMV CO-OPERATION DURING PATHOGENESIS}

There are several lines of evidence described above which suggest such GAPDH/OMV synergy:

- Both GAPDH and OMVs are secreted commonly (ubiquitously?) by pathogens.

- Both GAPDH and OMVs stimulate pathogenesis.

- GAPDH is a common component of OMVs.

- GAPDH is an adhesin, but also has membrane fusion activity.

- OMVs can deliver their contents beyond target membranes by fusing with them.

- GAPDH can enhance OMV activity by stimulating membrane fusion.

Pathogen-derived GAPDH has been shown to have a mechanistic role in tissue colonization and adherence, but in no other aspects of the pathogenicity of Gram-negative organisms. However, making a topological mutant that does not secrete GAPDH results in a strain with reduced (but not abolished) host cell adherence (Boël et al., 2005), indicating that pathogens have other adhesins that complement GAPDH's matrix-binding activity. Nevertheless, non-pathogenic strains of E. coli do not secrete GAPDH (Egea et al., 2007), which is taken as evidence that GAPDH is required for pathogenicity. Together these observations suggest that GAPDH has a role in virulence beyond just adhesion.

The few studies that have demonstrated membrane fusion by OMVs have taken no effort to reduce GAPDH levels/activity in their OMV preparations, and the organisms whose OMVs are known to fuse with membranes are also known to naturally contain GAPDH. GAPDH may be merely promoting an intrinsic OMV activity, but the possibly cannot be discounted that GAPDH is actually required for OMV membrane-fusion activity and resulting toxin delivery.

An interesting mechanistic feature common to OMV uptake and GAPDH-catalyzed membrane fusion is that both processes are thought to be dependent on specific lipids. The fusogenic activity of GAPDH requires cholesterol and the ether lipid plasmenylethanolamine, which are both commonly found in mammalian membranes (Glaser and Gross, 1995). Kesty et al.
(2004) showed that enterotoxigenic E. coli secretes enterotoxin via OMVs, and that host cells were able to endocytose the toxincontaining OMVs by a mechanism dependent on cholesterol-rich lipid rafts. In principle, GAPDH could stimulate OMVs to bind to cholesterol-rich membranes, which are then prime substrates for GAPDH-mediated fusion or host-mediated endocytosis (with delivery of OMV contents into the target cell).

There is also the potential for OMVs to affect GAPDH function reciprocally. OMVs increase the effective amount of bacterial OM, which GAPDH can cross-link by virtue of its properties as an adhesin, potentially promoting biofilm formation and uptake/fusion of OMVs.

\section{BEYOND PATHOGENESIS}

As OMVs and GAPDH appear to be ubiquitously secreted by Gram-negative bacteria, it is likely that GAPDH will be implicated in other functions of OMVs. Biofilm formation is an important and universal phenomenon, promoted by OMVs. It is also promoted by intercellular quorum signaling which itself can be transduced through OMVs (Mashburn and Whiteley, 2005). Mixed biofilms are frequently observed in nature, and competition between the different inhabitants is important for determining fitness. Delivery of toxins to competitors or prey organisms via OMVs has been observed and thus modulation of OMV activity by GAPDH would likely be an important fitness determinant.

In the laboratory, several obvious experiments arise from considering the potential involvement of GAPDH in OMV activity.

- No bacterial GAPDH has yet been shown to possess fusogenic activity and this needs to be confirmed, perhaps by monitoring lipid/content mixing through fluoresence quenching/enhancement (Glaser and Gross, 1994). Care would need to be taken however as GAPDH-mediated membrane fusion may be dependent on membrane lipid composition as it is in eukaryotes (Glaser and Gross, 1995).

- We would expect GAPDH-depleted OMVs to be impeded in their ability to fuse with target membranes. This would be a technically challenging prediction to test however, due to the important metabolic role of GAPDH precluding facile gene deletion, and the inherent membrane-binding affinity of GAPDH defying physical removal. Nevertheless, it should be possible to engineer a GAPDH deletant by developing appropriate media to support metabolic bypassing of glycolysis/gluconeogenesis in the mutant. Alternatively a "functional" mutant could be created by placing the GAPDH gene under the control of an inducible or repressible promoter, or through the creation of a topologically restricted version of GAPDH (Boël et al., 2005). GAPDH inhibitors are also available, which might also affect fusogenic activity [e.g., pentalenolactone and koningic (heptelidic) acid].

- GAPDH is expected to promote adhesion between bacterial cells, as well as cell-OMV adhesion. It would be interesting to see whether reducing membrane-associated GAPDH levels does impact negatively on colonial growth and/or aggregation. 
Perhaps by using simple assays which monitor sedimentation of aggregated cells, for example the approach used by Chang and Dworkin (1994) to measure bacterial cohesion.

- The effect of cholesterol and/or ether lipids on GAPDHmediated OMV-membrane fusion should be tested for a range of OMV producers and target membranes, to delineate any conservation of lipid requirements.

\section{IMPLICATIONS}

Bacterial GAPDH has already proven useful as a therapeutic target with the development of cross-protective GAPDH-based vaccines against Gram-negative and -positive bacteria for agriand aqua-culture (Li et al., 2011; Vanden Bergh et al., 2013; Velineni and Timoney, 2013; Trung Cao et al., 2014). The GAPDH inhibitor pentalenolactone (Cane and Sohng, 1994) is known to act as an antibiotic due to its disruption of bacterial glycolysis, but it is also potent against mammalian homologs and is not used in the clinic. Nevertheless, there are enough sequence differences between human and bacterial GAPDH to make GAPDH-targeted therapies for the clinic plausible (Seidler and Seidler, 2013), and such inhibitors could also be useful beyond the clinic as antibiofilm/antifouling compounds.

OMVs are proving efficacious as hapten components of antibacterial vaccines (Acevedo et al., 2014; Choi et al., 2014;

\section{REFERENCES}

Acevedo, R., Fernández, S., Zayas, C., Acosta, A., Sarmiento, M. E., Ferro, V. A., et al. (2014). Bacterial outer membrane vesicles and vaccine applications. Front. Immunol. 5:121. doi: 10.3389/fimmu.2014.00121

Alvarez, R. A., Blaylock, M. W., and Baseman, J. B. (2003). Surface localized glyceraldehyde-3-phosphate dehydrogenase of Mycoplasma genitalium binds mucin. Mol. Microbiol. 48, 1417-1425. doi: 10.1046/j.1365-2958.2003.03518.x

Bielaszewska, M., Aldick, T., Bauwens, A., and Karch, H. (2014). Hemolysin of enterohemorrhagic Escherichia coli: structure, transport, biological activity and putative role in virulence. Int. J. Med. Microbiol. 304, 521-529. doi: 10.1016/j.ijmm.2014.05.005

Boël, G., Jin, H., and Pancholi, V. (2005). Inhibition of cell surface export of group A streptococcal anchorless surface dehydrogenase affects bacterial adherence and antiphagocytic properties. Infect. Immun. 73, 6237-6248. doi: 10.1128/IAI.73.10.6237-6248.2005

Bomberger, J. M., Maceachran, D. P., Coutermarsh, B. A., Ye, S., O’Toole, G. A., and Stanton, B. A. (2009). Long-distance delivery of bacterial virulence factors by Pseudomonas aeruginosa outer membrane vesicles. PLoS Pathog. 5:e1000382. doi: 10.1371/journal.ppat.1000382

Cane, D. E., and Sohng, J. K. (1994). Inhibition of glyceraldehyde-3phosphate dehydrogenase by pentalenolactone. 2. Identification of the site of alkylation by tetrahydropentalenolactone. Biochemistry 33, 6524-6530. doi: 10.1021/bi00187a020

Chang, B. Y., and Dworkin, M. (1994). Isolated fibrils rescue cohesion and development in the Dsp mutant of Myxococcus xanthus. J. Bacteriol. 176, 7190-7196.

Choi, K. S., Kim, S. H., Kim, E. D., Lee, S. H., Han, S. J., Yoon, S., et al. (2014). Protection from hemolytic uremic syndrome by eyedrop vaccination with modified enterohemorrhagic E. coli outer membrane vesicles. PLoS ONE 9:e100229. doi: 10.1371/journal.pone.0100229

Curtis, P. D., Atwood, J. III, Orlando, R., and Shimkets, L. J. (2007). Proteins associated with the Myxococcus xanthus extracellular matrix. J. Bacteriol. 189, 7634-7642. doi: 10.1128/JB.01007-07
Nieves et al., 2014), and as adjuvants for delivery of heterologous haptens (Moshiri et al., 2012). Perhaps part of the success of OMV vaccines is because they are multivalent GAPDHpresenting entities. Rationally combining GAPDH and OMVs within vaccines has the potential to synergistically enhance immunogenicity of each component. It is plausible that OMVs could also see use in the clinic as antimicrobials. Not only have they been shown to kill bacteria directly but they can also act as delivery devices for antibiotics (Kadurugamuwa and Beveridge, 1998). Potentially, the addition of stimulatory "accessory proteins" such as GAPDH would help make such OMV-based approaches more effective.

Beyond the clinic, a holistic understanding of the interaction between GAPDH and OMVs will need to consider the relative physical location of both entities and modulators of their activities. This will be especially important when considering mixed communities of bacteria, expressing a range of OMVs and GAPDH isoforms with differing target specificities and fusogenic potential. However, an enhanced understanding of such processes will provide invaluable information regarding the mechanisms of bacterial competition and co-operation.

\section{AUTHOR CONTRIBUTIONS}

DW and BM conceived, drafted, and edited the work.
Deng, W., Yu, H. B., de Hoog, C. L., Stoynov, N., Li, Y., Foster, L. J., et al. (2012). Quantitative proteomic analysis of type III secretome of enteropathogenic Escherichia coli reveals an expanded effector repertoire for attaching/effacing bacterial pathogens. Mol. Cell. Proteomics 11, 692-709. doi: 10.1074/mcp.M111.013672

Dutson, T. R., Pearson, A. M., Price, J. F., Spink, G. C., and Tarrant, P. J. (1971). Observations by electron microscopy on pig muscle inoculated and incubated with Pseudomonas fragi. Appl. Microbiol. 22, 1152-1158.

Egea, L., Aguilera, L., Giménez, R., Sorolla, M. A., Aguilar, J., Badía, J., et al. (2007). Role of secreted glyceraldehyde-3-phosphate dehydrogenase in the infection mechanism of enterohemorrhagic and enteropathogenic Escherichia coli: interaction of the extracellular enzyme with human plasminogen and fibrinogen. Int. J. Biochem. Cell Biol. 39, 1190-1203. doi: 10.1016/j.biocel.2007.03.008

Ellis, T. N., and Kuehn, M. J. (2010). Virulence and immunomodulatory roles of bacterial outer membrane vesicles. Microbiol. Mol. Biol. Rev. 74, 81-94. doi: 10.1128/MMBR.00031-09

Elluri, S., Enow, C., Vdovikova, S., Rompikuntal, P. K., Dongre, M., Carlsson, S., et al. (2014). Outer membrane vesicles mediate transport of biologically active Vibrio cholerae cytolysin (VCC) from V. cholerae strains. PLoS ONE 9:e106731. doi: 10.1371/journal.pone.0106731

Evans, A. G. L, Davey, H. M., Cookson, A., Currinn, H., Cooke-Fox, G., Stanczyk, P. J. et al. (2012). Predatory activity of Myxococcus xanthus outer membrane vesicles and properties of their hydrolase cargo. Microbiology 158, 2742-2752. doi: 10.1099/mic.0.060343-0

Fahie, M., Romano, F. B., Chisholm, C., Heuck, A. P., Zbinden, M., and Chen, M. (2013). A non-classical assembly pathway of Escherichia coli pore-forming toxin cytolysin A. J. Biol. Chem. 288, 31042-31051. doi: 10.1074/jbc.M113.475350

Gao, J. Y., Ye, C. L., Zhu, L. L., Tian, Z. Y., and Yang, Z. B. (2014). A homolog of glyceraldehyde-3-phosphate dehydrogenase from Riemerella anatipestifer is an extracellular protein and exhibits biological activity. J. Zhejiang Univ. Sci. B 15, 776-787. doi: 10.1631/jzus.B1400023

Glaser, P. E., and Gross, R. W. (1994). Plasmenylethanolamine facilitates rapid membrane fusion: a stopped-flow kinetic investigation correlating the 
propensity of a major plasma membrane constituent to adopt an HII phase with its ability to promote membrane fusion. Biochemistry 33, 5805-5812. doi: 10.1021/bi00185a019

Glaser, P. E., and Gross, R. W. (1995). Rapid plasmenylethanolamine-selective fusion of membrane bilayers catalyzed by an isoform of glyceraldehyde3-phosphate dehydrogenase: discrimination between glycolytic and fusogenic roles of individual isoforms. Biochemistry 34, 12193-12203. doi: 10.1021/bi00038a013

Grenier, D., and Mayrand, D. (1987). Functional characterization of extracellular vesicles produced by Bacteroides gingivalis. Infect. Immun. 55, 111-117.

Henderson, B., and Martin, A. (2011). Bacterial virulence in the moonlight: multitasking bacterial moonlighting proteins are virulence determinants in infectious disease. Infect. Immun. 79, 3476-3491. doi: 10.1128/IAI. 00179-11

Hessler, R. J., Blackwood, R. A., Brock, T. G., Francis, J. W., Harsh, D. M., and Smolen, J. E. (1998). Identification of glyceraldehyde-3-phosphate dehydrogenase as a $\mathrm{Ca} 2+-$ dependent fusogen in human neutrophil cytosol. J. Leukoc. Biol. 63, 331-336.

Holland, C., Mak, T. N., Zimny-Arndt, U., Schmid, M., Meyer, T. F., Jungblut, P. R., et al. (2010). Proteomic identification of secreted proteins of Propionibacterium acnes. BMC Microbiol. 10:230. doi: 10.1186/1471-2180$10-230$

Ikemoto, A., Bole, D. G., and Ueda, T. (2003). Glycolysis and glutamate accumulation into synaptic vesicles. Role of glyceraldehyde phosphate dehydrogenase and 3-phosphoglycerate kinase. J. Biol. Chem. 278, 5929-5940. doi: 10.1074/jbc.M211617200

Inagaki, S., Onishi, S., Kuramitsu, H. K., and Sharma, A. (2006). Porphyromonas gingivalis vesicles enhance attachment, and the leucine-rich repeat BspA protein is required for invasion of epithelial cells by "Tannerella forsythia". Infect. Immun. 74, 5023-5028. doi: 10.1128/IAI.00062-06

Kadurugamuwa, J. L., and Beveridge, T. (1996). Bacteriolytic effect of membrane vesicles from Pseudomonas aeruginosa on other bacteria including pathogens: conceptually new antibiotics. J. Bacteriol. 178, 2767-2774.

Kadurugamuwa, J. L., and Beveridge, T. J. (1998). Delivery of the non-membranepermeative antibiotic gentamicin into mammalian cells by using Shigella flexneri membrane vesicles. Antimicrob. Agents Chemother. 42, 1476-1483.

Kadurugamuwa, J. L., and Beveridge, T. J. (1999). Membrane vesicles derived from Pseudomonas aeruginosa and Shigella flexneri can be integrated into the surfaces of other gram-negative bacteria. Microbiology 145(Pt 8), 2051-2060. doi: 10.1099/13500872-145-8-2051

Kamaguchi, A., Ohyama, T., Sakai, E., Nakamura, R., Watanabe, T., Baba, H., et al. (2003). Adhesins encoded by the gingipain genes of Porphyromonas gingivalis are responsible for co-aggregation with Prevotella intermedia. Microbiology 149, 1257-1264. doi: 10.1099/mic.0.25997-0

Kaneda, M., Takeuchi, K., Inoue, K., and Umeda, M. (1997). Localization of the phosphatidylserine-binding site of glyceraldehyde-3-phosphate dehydrogenase responsible for membrane fusion. J. Biochem. 122, 1233-1240. doi: 10.1093/oxfordjournals.jbchem.a021886

Kesty, N. C., Mason, K. M., Reedy, M., Miller, S. E., and Kuehn, M. J. (2004). Enterotoxigenic Escherichia coli vesicles target toxin delivery into mammalian cells. EMBO J. 23, 4538-4549. doi: 10.1038/sj.emboj.7600471

Kim, D. K., Kang, B., Kim, O. Y., Choi, D. S., Lee, J., Kim, S. R., et al. (2013). EVpedia: an integrated database of high-throughput data for systemic analyses of extracellular vesicles. J. Extracell. Vesicles 2:20384. doi: 10.3402/jev.v2i0.20384

Kulkarni, H. M., and Jagannadham, M. V. (2014). Biogenesis and multifaceted roles of outer membrane vesicles from Gram-negative bacteria. Microbiology 160, 2109-2121. doi: 10.1099/mic.0.079400-0

Li, X., Wu, H., Zhang, M., Liang, S., Xiao, J., Wang, Q., et al. (2011). Secreted glyceraldehyde-3-phosphate dehydrogenase as a broad spectrum vaccine candidate against microbial infection in aquaculture. Lett. Appl. Microbiol. 54, 1-9. doi: 10.1111/j.1472-765X.2011.03164.x

Manning, A. J., and Kuehn, M. J. (2013). Functional advantages conferred by extracellular prokaryotic membrane vesicles. J. Mol. Microbiol. Biotechnol. 23, 131-141. doi: 10.1159/000346548

Mashburn, L. M., and Whiteley, M. (2005). Membrane vesicles traffic signals and facilitate group activities in a prokaryote. Nature 437, 422-425. doi: $10.1038 /$ nature 03925
McBroom, A. J., and Kuehn, M. J. (2007). Release of outer membrane vesicles by Gram-negative bacteria is a novel envelope stress response. Mol. Microbiol. 63, 545-558. doi: 10.1111/j.1365-2958.2006.05522.x

Moshiri, A., Dashtbani-Roozbehani, A., Najar Peerayeh, S., and Siadat, S. D. (2012). Outer membrane vesicle: a macromolecule with multifunctional activity. Hum. Vaccin. Immunother. 8, 953-955. doi: 10.4161/hv. 20166

Nakagawa, T., Hirano, Y., Inomata, A., Yokota, S., Miyachi, K., Kaneda, M., et al. (2003). Participation of a fusogenic protein, glyceraldehyde-3-phosphate dehydrogenase, in nuclear membrane assembly. J. Biol. Chem. 278, 2039520404. doi: 10.1074/jbc.M210824200

Nieves, W., Petersen, H., Judy, B. M., Blumentritt, C. A., Russell-Lodrigue, K., Roy, C. J., et al. (2014). A Burkholderia pseudomallei outer membrane vesicle vaccine provides protection against lethal sepsis. Clin. Vaccine Immunol. 21, 747-754. doi: 10.1128/CVI.00119-14

Oliveira, L., Madureira, P., Andrade, E. B., Bouaboud, A., Morello, E., Ferreira, P., et al. (2012). Group B streptococcus GAPDH is released upon cell lysis, associates with bacterial surface, and induces apoptosis in murine macrophages. PLOS ONE 7:e29963. doi: 10.1371/journal.pone. 0029963

Pancholi, V., and Fischetti, V. A. (1992). A major surface protein on group A streptococci is a glyceraldehyde-3-phosphate-dehydrogenase with multiple binding activity. J. Exp. Med. 176, 415-426. doi: 10.1084/jem.176. 2.415

Pasztor, L., Ziebandt, A. K., Nega, M., Schlag, M., Haase, S., Franz-Wachtel, M., et al. (2010). Staphylococcal major autolysin (Atl) is involved in excretion of cytoplasmic proteins. J. Biol. Chem. 285, 36794-36803. doi: 10.1074/jbc.M110.167312

Roier, S., Blume, T., Klug, L., Wagner, G. E., Elhenawy, W., Zangger, K., et al. (2014). A basis for vaccine development: comparative characterization of Haemophilus influenzae outer membrane vesicles. Int. J. Med. Microbiol. 305, 298-309. doi: 10.1016/j.ijmm.2014.12.005

Rolhion, N., Barnich, N., Claret, L., and Darfeuille-Michaud, A. (2005). Strong decrease in invasive ability and outer membrane vesicle release in Crohn's disease-associated adherent-invasive Escherichia coli strain LF82 with the yfgL gene deleted. J. Bacteriol. 187, 2286-2296. doi: 10.1128/JB.187.7.22862296.2005

Seidler, K. A., and Seidler, N. W. (2013). Role of extracellular GAPDH in Streptococcus pyogenes virulence. Mo. Med. 110, 236-240. Available online at: https://www.kcumb.edu/uploadedFiles/Content/News/KCUMB_Role_Extrace llular_GAPDH_Streptoccoccus_Pyogenes_Virulence_MayJune2013_MoMed. pdf

Seidler, N. W. (2013). GAPDH and intermediary metabolism. Adv. Exp. Med. Biol. 985, 37-59. doi: 10.1007/978-94-007-4716-6_2

Sirover, M. A. (2005). New nuclear functions of the glycolytic protein, glyceraldehyde-3-phosphate dehydrogenase, in mammalian cells. J. Cell. Biochem. 95, 45-52. doi: 10.1002/jcb.20399

Sirover, M. A. (2014). Structural analysis of glyceraldehyde-3-phosphate dehydrogenase functional diversity. Int. J. Biochem. Cell Biol. 57, 20-26. doi: 10.1016/j.biocel.2014.09.026

Terrasse, R., Amoroso, A., Vernet, T., and Di Guilmi, A. M. (2015). Streptococcus pneumoniae GAPDH is released by cell lysis and interacts with peptidoglycan. PLoS ONE 10:e0125377. doi: 10.1371/journal.pone.0125377

Thay, B., Damm, A., Kufer, T. A., Wai, S. N., and Oscarsson, J. (2014). Aggregatibacter actinomycetemcomitans outer membrane vesicles are internalized in human host cells and trigger NOD1- and NOD2-dependent NF- $\mathrm{B}$ activation. Infect. Immun. 82, 4034-4046. doi: 10.1128/IAI. 01980-14

Trung Cao, T., Tsai, M. A., Yang, C. D., Wang, P. C., Kuo, T. Y., Gabriel Chen, H. C., et al. (2014). Vaccine efficacy of glyceraldehyde-3-phosphate dehydrogenase (GAPDH) from Edwardsiella ictaluri against E. tarda in tilapia. J. Gen. Appl. Microbiol. 60, 241-250. doi: 10.2323/jgam.60.241

Vanden Bergh, P., Heller, M., Braga-Lagache, S., and Frey, J. (2013). The Aeromonas salmonicida subsp. salmonicida exoproteome: global analysis, moonlighting proteins and putative antigens for vaccination against furunculosis. Proteome Sci. 11:44. doi: 10.1186/1477-5956-11-44

Vanhove, A. S., Duperthuy, M., Charrière, G. M., Le Roux, F., Goudenège, D., Gourbal, B., et al. (2015). Outer membrane vesicles are vehicles for the delivery 
of Vibrio tasmaniensis virulence factors to oyster immune cells. Environ. Microbiol. 17, 1152-1165. doi: 10.1111/1462-2920.12535

Velineni, S., and Timoney, J. F. (2013). Identification of novel immunoreactive proteins of Streptococcus zooepidemicus with potential as vaccine components. Vaccine 31, 4129-4135. doi: 10.1016/j.vaccine.2013.06.100

Wang, Y., Kim, S. G., Wu, J., Huh, H. H., Lee, S. J., Rakwal, R., et al. (2013). Secretome analysis of the rice bacterium Xanthomonas oryzae (Xoo) using in vitro and in planta systems. Proteomics 13, 1901-1912. doi: 10.1002/pmic.201200454

Whitworth, D. E. (2011). Myxobacterial vesicles: death at a distance? Adv. Appl. Microbiol. 75, 1-31. doi: 10.1016/B978-0-12-387046-9.00001-3

Whitworth, D. E., Slade, S. E., and Mironas, A. (2015). Composition of distinct subproteomes in Myxococcus xanthus: metabolic cost and amino acid availability. Amino Acids. doi: 10.1007/s00726-015-2042-x. [Epub ahead of print].
Yonezawa, H., Osaki, T., Kurata, S., Fukuda, M., Kawakami, H., Ochiai, K., et al. (2009). Outer membrane vesicles of Helicobacter pylori TK1402 are involved in biofilm formation. BMC Microbiol. 9:197. doi: 10.1186/1471-2180-9-197

Conflict of Interest Statement: The authors declare that the research was conducted in the absence of any commercial or financial relationships that could be construed as a potential conflict of interest.

Copyright $\odot 2015$ Whitworth and Morgan. This is an open-access article distributed under the terms of the Creative Commons Attribution License (CC BY). The use, distribution or reproduction in other forums is permitted, provided the original author(s) or licensor are credited and that the original publication in this journal is cited, in accordance with accepted academic practice. No use, distribution or reproduction is permitted which does not comply with these terms. 